ROCZNIKI HUMANISTYCZNE

Tom LXVIII, zeszyt 8 - 2020

DOI: http://dx.doi.org/10.18290/rh.20688-6

BEATA JEZIERSKA

\title{
LA VALEUR SÉMANTIQUE AJOUTÉE ET PERDUE DANS LA TRADUCTION EN ITALIEN DU DRAME POLONAIS PRZED SKLEPEM JUBILERA DE KAROL WOJTYŁA
}

\author{
SEMANTIC VALUE ADDED AND LOST IN THE ITALIAN TRANSLATION \\ OF THE POLISH DRAMA PRZED SKLEPEM JUBILERA BY KAROL WOJTYŁA
}

Abstract

The aim of the article is to describe a phenomenon of value added and lost at the lexical and semantic level in the literary translation. The study is based on the analysis of the parallel PolishItalian text of the drama Przed sklepem jubilera [The jeweller's shop] by Karol Wojtyła. The comparison of the original Polish text with its Italian translation made it possible to distinguish semantic nuances lost at the lexical level of the translation, while the results of the reverse operation (comparison of the target text in Italian with the source text in Polish) prove the presence of many "added" lexical elements in the final text. They are mostly multi-word combinations with varying degrees of idiomaticity, i.e. phraseological units. Traces of semantic losses as well as added elements clearly indicate a creative role of the literary translator who becomes in this way the "second author" of a text.

Key words: literary translation; lexical choices; phraseological units; parallel text.

La lecture parallèle de la traduction et du texte original garantit presque toujours l'acquisition d'un matériel linguistique intéressant, surtout quand il s'agit de textes artistiques. Il existe deux types de stratégies qui peuvent être appliquées dans ce cadre : nous regardons le texte original et vérifions comment les éléments sélectionnés ont été traduits dans la langue cible ou nous

Docteur BEATA JEZIERSKA - Université d’Udine, Italie (Università degli Studi di Udine, Dipartimento di lingue e letterature, comunicazione, formazione e società) ; doctorat en linguistique polonaise à UAM (2016) ; e-mail : beata.jezierska@uniud.it. ORCID: https://orcid.org/00000002-0444-3285.

Dr Beata Jezierska - Uniwersytet w Udine, Włochy (Università degli Studi di Udine, Dipartimento di lingue e letterature, comunicazione, formazione e società), doktorat $\mathrm{z}$ językoznawstwa polskiego na UAM (2016); e-mail: beata.jezierska@uniud.it. ORCID: https://orcid.org/00000002-0444-3285. 
analysons d'abord le texte de traduction pour ensuite découvrir la source des usages linguistiques qui nous intéressent. Ces deux activités ont des objectifs différents, bien qu'ils soient complémentaires en général. Dans le premier cas, nous nous concentrons sur la pratique, c'est-à-dire sur les méthodes de traduction et sur l'aspect contrastif (pour répondre à la question de base : comment un élément X d'une langue A est-il traduit vers une langue B), tandis que dans le second cas l'accent est mis sur la recherche de traces de la « présence » du traducteur qui devient « deuxième auteur ${ }^{1}$ du texte : nous analysons ce qui a été ajouté (ou changé) par le traducteur dans le texte d'arrivée au regard du texte original. De cette façon, en exposant la figure du traducteur dans le processus de traduction, nous prouvons son rôle créatif (et non reproductif); par conséquent, la traduction elle-même est considérée comme " une nouvelle valeur culturelle dans un nouveau contexte de la (...) langue nationale $»^{2}$ (Pieńkos 82 ) et pas seulement une copie du texte traduit.

Cet article aborde l'analyse axée sur l'observation des choix lexicaux et phraséologiques dans la traduction italienne du texte polonais de la pièce de théâtre intitulée Przed sklepem jubilera (en italien : Bottega dell'orefice) ${ }^{3}$. Karol Wojtyła l'avait publiée en 1960 dans la revue catholique Znak ('le Signe') sous le pseudonyme d'Andrzej Jawień ${ }^{4}$. À ce moment-là, il était évêque auxiliaire de Cracovie, donc il avait déjà une certaine position dans les structures de l'Église ; cependant, il restait toujours un homme de littérature, de poésie et de théâtre. Dans cette pièce courte et condensée, qui fait partie d'une trilogie dramatique $^{5}$, le futur pape et saint de l'Église catholique reprend le grand sujet de l'amour des amoureux et puis, des époux. Il le fait avec sa propre sensibilité et perspicacité qui étonne vraiment et touche; avec fascination ${ }^{6}$ et en couvrant l'ensemble de l'expérience profondément humaine. Ses personnages dramatiques sont aux prises avec l'amour, spirituel et sensuel; ils le rejettent souvent, comme André, mari de Thérèse et père de Christophe :

\footnotetext{
${ }^{1}$ Dans la tradition polonaise, ce terme a été introduit en traductologie par Anna Legeżyńska.

${ }^{2}$ Toutes les citations de sources polonaises ou italiennes sont fournies dans ma propre traduction

${ }^{3}$ Titre en français : La Boutique de l'Orfèvre.

${ }^{4}$ Pour en savoir plus sur ce pseudonyme, voir l'avant propos de Jean Offredo dans l'édition française de l'œuvre en question (Wojtyła, La Boutique de l'Orfèvre 9).

${ }^{5}$ Avec d'autres titres : Brat naszego Boga, écrit dans les années 1944-1950 (Frère de notre Dieu), et Promieniowanie ojcostwa, écrit en 1964 (Rayonnement de la paternité).

${ }^{6}$ Agnieszka Komorowska (218) cite dans ce contexte une déclaration du pape lui-même : «Quand j'étais jeune prêtre, j'ai appris à aimer l'amour humain. C'était un des thèmes sur lesquels j'ai axé tout mon sacerdoce, mon ministère dans la prédication, au confessionnal et à travers ce que j'écrivais » (ici selon l'édition française de l'essai Entrez dans l'Espérance (Jean Paul II 192).
} 
L'amour, je voulais le croire, était une passion,

qui vous domine,

qui tient de l'absolu.

Je n'arrivais donc pas à comprendre

d'où venait cette étrange persistance,

cette constante présence de Thérèse (...).

J'avais l'impression d'être persécuté par mon amour,

je voulais y couper court, énergiquement.

L'amour se nourrit-il d'opposition ?

Est-il un affrontement (...) ? (KW-FR 15)

Cet auvre est une pièce de théâtre, mais il ne comporte pas d'actions, d'événements ou de dialogues classiques. Ce sont plutôt des monologues exposant les mêmes faits du point de vue de personnes différentes; d'après Stefan Sawicki (450) : «Tout se passe dans les souvenirs, les prémonitions, les rêves. La réalité perd des dimensions réalistes, prend une signification ambiguë et symbolique $»^{7}$. L'espace principal dans lequel «le spectacle » est projeté se trouve juste «devant» la boutique d'un vieil orfèvre, à sa devanture, dans la rue (et ce mot: devant a été omis dans les traductions française et italienne). C'est ici, dans ce «lieu», que les réflexions de tous les personnages du drame «se rencontrent » et forment une mosaïque qui sert à découvrir systématiquement un mystère du sacrement du mariage, du sens de la vie conjugale et de l'amour des conjoints. Ce dernier, selon l'analyse de l'image linguistique de l'amour dans le drame de Wojtyła, est considéré comme « la relation physique et spirituelle, participation à la vie de son conjoint, aide mutuelle sans attendre d'avantages (échange de dons), le désir de bonheur pour son époux, respect et la durabilité $»^{8}$ (Kopeć 39).

L'atmosphère intime du drame est construite sur une certaine simplicité de l'expression linguistique qui rend ces «méditations » (comme indiqué dans le sous-titre du drame) encore plus «humaines », proches de l'expérience de chaque homme qui aime ou pense aimer quelqu'un dans sa vie. En ce qui concerne le texte original en polonais, c'est surtout une syntaxe simple (phrases relativement courtes, toujours terminées) qui reste en harmonie avec le lexique précisément choisi, cependant riche en synonymes et en mots d'origine littéraire ou biblique. Ce qui attire l'attention au niveau lexical, c'est le fait que le

\footnotetext{
${ }^{7}$ En polonais : « Wszystko dzieje się we wspomnieniach, przeczuciach, marzeniach. Rzeczywistość zatraca realistyczne wymiary, nabiera niejednoznacznej, symbolicznej wymowy ».

${ }^{8}$ En polonais : «więź fizyczna i duchowa, współuczestniczenie w życiu drugiej osoby, wzajemna bezinteresowna pomoc (wymiana darów), pragnienie szczęścia drugiej osoby oraz szacunek i trwałość ».
} 
texte est presque privé d'unités phraséologiques. Enfin, il ne s'agit pas de montrer toutes ses capacités rhétoriques ; Wojtyła obtient une charge poétique désirée d'une manière différente, à travers des images (l'amour devient ainsi un chemin commun, parfois difficile, une lampe allumée qui donne de la lumière et chaleur, une source de vie, un contenant ouvert et une navette du tisserand - cf. Kopeć 38). Les traducteurs de l'œuvre devaient faire face à ce type de l'expression littéraire. Le drame a été traduit vers l'italien par Aleksandra Kurczab et Jerzy Pomianowski, un couple d'artistes et intellectuels polonais restant en exil en Italie pendant de nombreuses années (ils ont émigré suite à la vague d'antisémitisme amorcée après les événements de mars 1968 en Pologne communiste). Le texte italien, par rapport à la version originale du drame, constitue la base de l'observation dans cet article.

\section{TEXTE ORIGINAL FACE A LA TRADUCTION}

Comme déjà indiqué, mon objectif principal consiste à l'analyse des choix lexicaux dans le texte cible à travers le prisme du texte source. Je vais donc m'arrêter sur les éléments lexicaux et phraséologiques qui existent dans le texte original polonais et qui ne sont pas représentés « littéralement » dans le texte italien, afin de répondre à la question de recherche sur la valeur sémantique ajoutée et perdue au niveau lexical dans le processus de traduction. Ce sont des aspects très délicats de l'équivalence, un problème central de la traductologie, généralement défini comme une "relation d'identité établie dans le discours entre des unités de traduction de langues différentes, dont la fonction discursive est identique ou presque identique» (Delisle et al. 36). Les efforts visant à obtenir l'équivalence peuvent être considérés de manière globale comme une stratégie d'une traduction spécifique, en référence au texte entier, d'après Delisle et al. (77) : «selon le cas, le traducteur peur adopter une stratégie d'adaptation ou de traduction littérale, changer le genre d'un texte ou le modifier ». Par contre, dans cet article, je me concentre sur les micro-contextes et l'analyse des exemples collectés par rapport aux procédés de traduction ${ }^{9}$ individuels et les résultats de l'application de ces procédés, connus et décrits dans la littérature traductologique. Je m'intéresse ainsi aux questions : 1) de la forme de l'équivalence et 2) de la relation des

\footnotetext{
${ }^{9}$ Selon Delisle et al. (64) : «Par opposition aux stratégies de traduction qui orientent la démarche globale du traducteur à l'égard du texte à traduire, les procédés de traduction portent sur des segment de texte relevant du microcontextes ».
} 
éléments lexicaux ajoutés ou soustraits dans le texte cible au regard de la notion de l'équivalence.

Quelques observations principales peuvent être données quant au texte parallèle du drame de Wojtyła en polonais et en italien. Ce qui attire immédiatement l'attention, ce sont des traitements liés aux noms propres. Tous les personnages individuels ont des prénoms traditionnels, présents dans les plus anciens textes de la culture européenne. Certains d'entre eux ont les formes identiques en deux langues qui nous intéressent (PL Teresa - IT Teresa, PL Anna - IT Anna) ou presque identiques (PL Monika - IT Monica, PL Stefan - IT Stefano, PL Adam - IT Adamo) ; au contraire, dans deux cas les différences graphiques et morphologiques sont importantes et la conservation des formes originales polonaises Krzysztof (IT Cristoforo) ou Andrzej (IT Andrea) dans le texte cible ne les rendrait pas lisibles en tant que universelles (traditionnelles) pour un lecteur italien. Cette adaptation semble donc une bonne solution.

Au-delà de l'adaptation évidente des noms de personnages, on y trouve d'autres exemples des procédés particuliers concernant les noms propres, comme ci-dessous :

PL Ten sam głos powtórzył się raz jeszcze, wówczas chłopcy zdecydowali się odkrzyknąć.

Przez cichy uśpiony las, przez noc bieszczadzką szedł sygnał. (KW-PL 14)

IT La stessa voce si udì un'altra volta.

Allora i ragazzi risposero, il loro richiamo attraversò la foresta muta e assopita,

la notte delle montagne. (KW-IT 12)

Dans le texte original nous avons donc "la nuit des Bieszczady » (noc bieszczadzka - le mot bieszczadzka, au nominatif : bieszczadzka, est un dérivé adjectif de nom géographique Bieszczady), tandis que les traducteurs ont décidé de ne laisser que « la nuit de la montagne » (la notte delle montagne). Il en résulte une certaine perte au plan de l'imagerie poétique; on sait que Wojtyła aimait les montagnes et les connaissait bien; par conséquent, ce n'est pas par hasard qu'il indique exactement cette chaîne de montagnes dans son texte. Bieszczady est le nom polonais de la partie orientale des Beskides, des massifs montagneux des Carpates s'étendant le long de la frontière entre la Pologne et la Slovaquie. Ils sont nettement plus bas que les Tatras, les montagnes plus populaires, «touristiques », uniques en Pologne qui 
offrent des paysages alpins. En revanche, les Bieszczady sont une destination en-dehors des sentiers battus, appréciée pour sa nature intacte et son caractère plus sauvage. En supprimant cette référence dans le texte cible italien, les traducteurs ont rendu l'image poétique plus universelle. Pareillement, ils ont décidé de supprimer les noms de rue dans le passage suivant (Chłodna, Prusa) :

PL Idę tym samym krawężnikiem, jak chodziłam, gdy byłam małą dziewczynką. Umiałam wówczas biec po krawężnikach ulic i nigdy noga nie spadła poniżej - na jezdnię. To była ulubiona zabawa koleżanek, po której przekomarzałyśmy się nieraz: ,a ja przebiegłam Chłodną i Prusa, i raz tylko spadłam”, ,a ja ani razu, widzisz, kto lepiej”. (KW-PL 51)

IT Cammino proprio sul ciglio come facevo quando ero piccola. Sapevo allora percorrere la lunga fila di pietre senza mai cadere, mai scivolare sul selciato. Questo era il nostro gioco preferito quando con le mie amichette ci rimbeccavamo più volte: "Io ho percorso la strada fino in fondo, e solo una volta sono scivolata", "E io nemmeno una volta, vedi chi è la più brava..." (KW-IT 48)

Il est vrai que ces noms de rue dans la version originale ne permettent pas nécessairement de localiser des événements du drame dans un endroit spécifique d'une ville particulière, mais tout de même, ils les situent en Pologne. Il faut cependant admettre que pour l'ensemble du texte l'aspect de l'insertion des événements du drame en Pologne n'est pas le plus important, parce que les choses pourraient avoir lieu n'importe où, dans n'importe quelle rue (la strada) du monde. En polonais, ces sont deux rues spécifiques et nommées (Chtodna et Prusa $^{10}$ ), en italien les filles courent « tout au long» (fino in fondo) une seule rue. C'est encore un autre exemple de l'adaptation dont la fonction supplémentaire est de souligner la valeur universelle de l'image poétique.

La langue polonaise a pour un trait caractéristique important la présence de séries de synonymes complets ; souvent ces éléments synonimiques ne diffèrent que par l'étymologie : slave ou latin, p.ex. chwała - sława - gloria pour 'gloire', młodość - adolescencja pour 'adolescence', obszar - terytorium pour 'territoire', uszkodzenie - wada - defekt pour 'défaut', wielbiciel - adorator pour 'adorateur', zgoda - akcteptacja pour 'acceptation' etc. (cf. WSWO). Parfois, dans les pairs de synonymes, les lexèmes s'opposent par

\footnotetext{
${ }^{10} \mathrm{Du}$ point de vue grammatical, la forme Chłodna 'froide' est un adjectif féminin et fonctionne en polonais surtout comme un mot commun ; Prusa c'est le génitif du nom propre Prus, il s'agit d'un écrivain célèbre polonais Bolesław Prus.
} 
des nuances stylistiques, c'est-à-dire l'un d'eux a une valeur neutre, tandis que l'autre est fortement marqué (littéraire). Ainsi, dans le texte source polonais de Wojtyła on trouve le mot zwierciadto ('miroir') auquel correspond un simple équivalent italien lo specchio :

PL Pamiętam - okno tego sklepu niegdyś stało się dziwnym zwierciadłem, chłonęło naszą przyszłość aż do tego momentu, w którym zaczynała się tajemnica. (KW-PL 81)

IT Ricordo - la vetrina di questo negozio una volta divenne uno specchio strano, assorbì tutto il nostro futuro, fino al momento in cui inizia il mistero. (KW-IT 78)

ou encore:

PL Nie było już nawet zwierciadła, w którym kiedyś oboje z Andrzejem ujrzeliśmy przyszłość najbliższą. (KW-PL 87)

IT Lo specchio nel quale una volta vedemmo con Andrea il nostro futuro - non c'era più. (KW-IT 83)

L'auteur utilise ce mot d'origine slave (zwierciadto) d'une manière conséquente et le fait juste en raison de ses propriétés stylistiques : en polonais contemporain, c'est un lexème plus poétique, sublime et rare par rapport à son analogue neutre d'origine latine lustro (cf. ETYM). Dans la version italienne, ce détail est devenu invisible et a été perdu.

Le même mécanisme s'est produit en cas de traduction en italien d'un verbe typiquement slave miłować 'aimer', étroitement lié au substantif miłość ('amour'), mais autrefois plus proche sémantiquement de la charité (polonais taska) et la miséricorde (polonais mitosierdzie) :

PL Słyszałabyś wtedy, jak mówi: umiłowana, nie wiesz, jak bardzo należysz do mnie, jak bardzo jesteś własnością mojej miłości i mego cierpienia - bo „milować” znaczy dawać życie poprzez śmieré, „milować” znaczy wytryskać zdrojem wody żywej w głębinach duszy, która się pali i tli, a spłonąć nie może. (KW-PL 56)

IT Sentiresti allora il richiamo: oh, mia amata, tu non sai quanto mi appartieni, non sai quanto appartieni al mio amore a alla mia pena - perché amare vuol dire donare la vita attraverso la morte, amare vuol dire sprigionare della profondità dell'anima l'acqua viva della sorgente, l'anima che brucia, arde senza fiamma, ma non riesce a ridursi in cenere. (KW-IT 53) 
Aujourd'hui, le lexème miłować est associé au registre soutenu de la langue polonaise (SJP, WSJP). Par contre le verbe synonyme kochać, d'origine slave aussi et étymologiquement connecté au morphème sémantique slave kos-, koch- 'toucher' (cf. ETYM), reste plus universel et couvre une multitude d'aspects de l'amour : spirituel, émotionnel et sensuel. Il est représenté sur les différents niveaux de langue et n'est pas associé à un registre particulier. Dans le passage cité plus haut, Wojtyła fait une référence claire à la parabole de dix vierges et au concept du mariage du Christ avec son Église, donc le mot miłować s'harmonise d'autant plus avec le sujet. Cependant, le lexème italien amare semble un pur équivalent du polonais kochać et pour cette raison on peut dire que l'on perde dans le texte traduit ce nuance sémantique de la sublimité de l'expérience religieuse. Il est intéressant de remarquer que l'italien dispose de deux espressions pour exprimer deux notions différentes contenues dans de ce que l'on exprime en polonais par le verbe kochać et en français par aimer: la phrase ti amo 'je t'aime' implique l'amour " plein », y compris l'amour romantique et sensuel, tandis que l'expression $t i$ voglio bene fait signe vers la bienveillance (la benevolenza), le latin caritas, c'est-à-dire l'amour du prochain (voir tableau ci-dessous).

\begin{tabular}{|c|c|c|}
\hline polonais & italien & français \\
\hline kocham cię & ti amo & je t'aime \\
\cline { 2 - 2 } & ti voglio bene & \\
\hline
\end{tabular}

Dans la traduction italienne du drame la construction voler bene a qualcuno n'apparaît qu'une seule fois :

PL Byłem przejęty jej wzruszeniem, a pośrednio moim przeżyciem jej wzruszenia, bo odebrałem je w pełni - i zobaczyłem nas dwoje na dnie tych naszych przeżyć: ja chyba bardzo ją kocham. (KW-PL 76)

IT Ero tutto preso dalla sua commozione e da come la vivevo io stesso, perché la sentivo pienamente - e ho visto noi due sul fondo di questa esperienza; credo di volerle molto bene. (KW-IT 73)

Le sujet de tels changements sémantiques entre les langues mérite certainement une étude plus approfondie.

Le personnage introduit par Wojtyła dans ce passage, qui fait référence au récit biblique de dix vierges, c'est l'Époux : Oblubieniec en polonais et lo 
Sposo en italien. Les deux noms, polonais et italien, sont conformes aux traditions de traductions de la Bible dans ces deux langues. Cependant, le nom polonais Oblubieniec ('fiancé, jeune marié') est marqué (ancien - SJP) ; en italien le lexème lo sposo n'est pas limité au registre littéraire de la langue et reste « neutre» stylistiquement.

Un phénomène similaire peut être observé dans le passage suivant :

PL Ongiś małą dziewczynkę prowadzili oboje za rączki,

a jeszcze dawniej byłaś niemowlęciem

i ojciec twój wracał po pracy

i pytał matki twej, Anny,

czy przybrałaś na wadze, Moniko, i czy masz dobry apetyt,

i cieszył się każdym gramem twojego małego ciałka (...). (KW-PL 79)

IT Un tempo ti tenevano tutti e due per mano,

prima ancora eri nella culla,

e quando tuo padre tornava dal lavoro

e domandava ad Anna, tua madre:

- Come mangia la piccola, è aumentata di peso?

- e si congratulava per ogni grammo di più (...) (KW-IT 75)

Le lexème ongiś ('jadis') est qualifié par les dictionnaires du polonais contemporain comme ancien ou rare (SJP, WSJP); la forme neutre dans chaque registre est : kiedyś ('autrefois'). Cet aspect disparaît dans la traduction italienne qui utilise un lexème stylistiquement neutre un tempo.

Un autre type de perte sémantique résulte de l'omission d'un élément du sens du texte polonais :

PL Dzisiaj więc, gdy Andrzej zapytał: „czy zechciałabyś zostać na zawsze towarzyszką mojego życia?” ja po upływie dziesięciu minut odpowiedziałam „tak” (...). (KW-PL 13)

IT Oggi dunque, quando Andrea mi domandò: "Vuoi essere la compagna della mia vita?" dopo dieci minuti li risposi di sì (...). (KW-IT 15)

Dans la version originale Andrzej demande à Teresa si elle veut être sa compagne pour la vie (la compagnia della mia vita - towarzyszka mojego życia), pour toute la vie, pour toujours (na zawsze). Il est difficile à dire pourquoi les traducteurs ont omis cette expression.

En résumé, la comparaison du texte original polonais avec sa traduction italienne a permis de distinguer des nuances sémantiques perdues au niveau lexical. Ce sont des différences délicates et irrégulières, intéressantes du point de vue de la valeur sémantique, aussi dans le cadre d'études contrastives. 


\section{TEXTE DE TRADUCTION FACE AU TEXTE ORIGINAL}

Comme nous l'avons signalé au début, le texte polonais du drame de Wojtyła est presque dépourvu d'unités phraséologiques; on y trouve juste quelques expressions courantes tel que : poprosić kogoś o rękę [demander la main de quelqu'un] $]^{11}$ au sens de 'demander une femme en mariage' (PSFBiL), stanać $w$ miejscu [rester immobile] - 'ne pas progresser, ne pas se développer' (WSF), w każdym razie [en tout cas] - 'après tout, cependant', za wszelka cene [à tout prix] - 'quel que soit le coût ou l'effort engagé; nécessairement' (WSF), z biegiem czasu [avec la course du temps] - 'au fil du temps' (SJPD), zwrócić uwage na kogoś, coś [tourner l'attention vers quelqu'un ou quelque chose] - 'remarquer, s'intéresser à quelqu'un, quelque chose' (WSF). Ils sont traduits dans la version italienne du drame par : 1) un seul mot ou une séquence libre ${ }^{12}$ (PL stanać w miejscu - IT fermarsi, PL zwrócić uwage na kogoś, coś - IT guardare, PL z biegiem czasu - IT col tempo) ou 2) un équivalent phraséologique simple ${ }^{13}$ (PL poprosić kogoś o ręke - IT chiedere la mano di qualcuno, $\mathrm{PL} w$ każdym razie - IT in ogni caso, PL za wszelka cene - IT a qualsiasi costo).

En revanche, si nous observons la traduction italienne du texte de Wojtyła (pour révéler l'arrière-plan d'origine à l'étape suivante), nous constatons un nombre plus important d'éléments phraséologiques: l'analyse a permis d'identifier 45 unités linguistiques figées. C'est un phénomène très intéressant qui confirme les propriétés spécifiques des unités phraséologiques et leurs diverses fonctions dans la traduction littéraire. Dans ce cadre, l'une de leurs caractéristiques les plus importantes est la pictorialité «capacité à évoquer des représentations et des images dans l'esprit », mentionné par Bogusławski dans son article de synthèse consacré aux problèmes de propriétés d'unités phraséologiques ${ }^{14}$ (cf. Bogusławski).

\footnotetext{
${ }^{11}$ Entre les crochets carrés : la traduction mot à mot.

${ }^{12} \mathrm{C}$ 'est-à-dire une combinaison de mots sans aucune trace du figement.

${ }^{13}$ Par un équivalent simple (sémantique et structural) on entend une unité phraséologique de structure lexicale et de signification identiques dans deux langues (p.ex. IT ammazzare il tempoFR tuer le temps 'échapper à l'ennui en s'occupant ou en se distrayant' DEL ; IT aprire gli occhi a qualcuno - FR ouvrir les yeux à quelqu'un 'lui montrer ce qu'il se réfusait à voir, lui révéler quelque chose' DEL; IT testa calda - FR tête chaude 'exalté, qui se jette dans des aventures dangereuses' DEL). Par contre, un équivalent sémantique constitue une combinaison lexicale différente dans la deuxième langue ( $\mathrm{p}$. ex. IT comprare a scatola chiusa - FR acheter chat en poche 'acheter ou recevoir quelque chose sans vérifier sa nature ou son état' DEL ; IT avere la testa fra le nuvole - FR être dans la lune 'être distrait, rêver' DEL).

${ }^{14} \mathrm{La}$ terminologie et les définitions de cette notion diffèrent sans doute entre les pays et les
} 
Selon une étude sur le statut de l'unité phraséologique dans le texte cible de la traduction littéraire (réalisée sur un corpus français-polonais avec plus de 3000 couples de mots), la moitié des cas du choix de traduction par une unité phraséologique relève d'un caractère créatif et constitue ainsi une claire "valeur ajoutée » du texte ${ }^{15}$ (Jezierska, "Status of phraseological units »). Ce sont surtout des exemples dans lesquels une séquence figée présente dans le texte d'arrivée n'est pas un reflet dans le texte de départ. Dans le texte original on trouve deux types de phénomènes : 1) un seul lexème ou 2) un «zéro », à savoir aucune unité lexicale qui pourrait correspondre à une unité phraséologique utilisée par le traducteur.

Le premier type de ce phénomène est représenté dans la traduction du drame de Wojtyła. Les exemples incluent de nombreux cas simples (IT prendere la parola 'commencer un discours, en réunion ou en public' (TRE) à la place du verbe PL przemówić 'parler' ou bien IT man a mano 'progressivement' au lieu de PL powoli 'lentement, graduellement'), y compris quelques locutions figées d'usage relativement fréquent dans la langue tel que venire in mente 'idées qui apparaissent soudainement' (TRE), ce qui correspond dans le texte original au verbe pomyśleć 'penser' :

IT Allora mi vennero in mente le fedi che ancora portiamo al dito io e lui. (KW-IT 37)

PL Pomyślałam wtedy o obrączkach, które nosimy na palcach oboje: Stefan i ja. (KW-PL 40)

Le second phénomène (pas d'équivalent dans le texte source) se produit dans le passage suivant, dans lequel la locution verbale fare le ore piccole 'rentrer chez soi ou se coucher après minuit' (COR) a été sans doute ajoutée par les traducteurs :

traditions philologiques locales (cf. Beata Jezierska, 2016 : 13-46 ; 2019) ; dans la linguistique polonaise (qui développe continuellement des études phraséologiques du polonais depuis les années 1960) l'unité phraséologique est reconnu en général comme une combinaison fixe de deux ou plusieurs mots dont la signification n'est pas dérivée de la signification des composants, c'està-dire, en d'autres termes : "n'équivale pas simplement à la somme des significations des éléments » (Bally 74).

${ }^{15}$ Par contre, dans d'autres cas, ce sont différentes formes d'équivalence caractérisées par un certain automatisme. 
IT Perché il nostro vecchio orefice non spegne la luce? Invece di fare le ore piccole, meglio chiudere e andare a casa. (KW-IT 29)

PL Dlaczego świeci się jeszcze w pracowni starego jubilera? $\varnothing$ Powinien też już zamknąć i wrócić do domu. (KW-PL 32)

D'ailleurs, en termes de la structure de l'énoncé, on peut observer ici une modulation ${ }^{16}$ par un changement de catégorie grammaticale : dans la version polonais "la lumière est encore allumée », en italien c'est "l'orfèvre (qui) n'éteint pas la lumière».

Dans l'exemple suivant, il y a une coïncidence : les traducteurs ont utilisé une locution figée qui correspond dans le texte original à une séquence libre de forme identique en termes de composition lexicale :

IT Credimi, Andrea, mi sono balzati agli occhi i contrasti che dormono dentro di te. (KW-IT 20)

PL I wierz mi - prawie skoczyly mi do oczu te dysproporcje, które w tobie drzemią. (KW-PL 22)

La locution italienne saltare, balzare agli occhi 'être très évident, se démarquer avec une grande clarté, généralement utilisé pour des choses qui auraient dû rester cachées ; pour les défauts, les erreurs etc.' (COR) reflète fidèlement le sens de la phrase de départ qui n'a pas de caractéristiques idiomatiques. D'ailleurs, en polonais, il existe une unité phraséologique de composition lexicale similaire à celle italienne : skakać komuś, sobie (wzajemnie) do oczu, mais de signification différente 'se disputer vivement' (WSF).

Un autre traitement intéressant peut être vu ci-dessous :

IT Convinti che hanno toccato il settimo cielo dell'amore - non lo hanno sfiorato nemmeno. (KW-IT 45)

PL Porwani myślą, że wchłonęli całą tajemnicę miłości, a tymczasem nawet jeszcze jej nie dotknęli. (KW-PL 48)

C'est une combinaison de deux variantes phraséologiques de la même signification : essere al settimo cielo et toccare il cielo con un dito 'obtenir un maximum de bonheur et de félicité, montrer une grande joie' (COR) qui a été appliquée pour traduire une séquence libre d'une charge poétique importante

${ }^{16}$ Défini comme : «procédé de traduction qui consiste à restructurer un énoncé du texte d'arrivée en faisant intervenir un changement de point de vue ou d'éclairage par rapport à la formulation originale, ce qui se produit, notamment, quand on emploie la partie pour le tout, l'abstrait pour le concret, l'actif pour le passif » (Delisle et al., 1999 : 54). 
(dans la version polonaise, les deux amoureux pensent « d'avoir absorbé tout le secret de l'amour »). On peut donc conclure que la ressource phraséologique de la langue d'arrivée devient particulièrement utile dans le processus de traduction en cas de condensation de sens dans les textes littéraires.

\section{CONCLUSIONS}

L'objectif de l'analyse effectuée dans cet article était de trouver et de caractériser des éléments sémantiques ajoutés ou perdus dans la traduction littéraire. Il s'agit des décisions conscientes du traducteur qui sont à l'origine des choix lexicaux; nous ne traitons pas d'erreurs (résultant généralement d'une action inconsciente). De cette façon, en exposant la nature intentionnelle des solutions de traduction, nous démontrons la subjectivité du traducteur et son rôle créatif plutôt que reproductif dans le processus qui nous intéresse. On peut dire que le traducteur d'une œuvre littéraire dispose d'un champ de compétences d'auteur et devient ainsi "le deuxième auteur », parce qu'il est responsable de l'introduction de la valeur artistique dans un nouveau contexte linguistique et culturel. C'est un défi sérieux, nécessitant non seulement du talent littéraire mais aussi des rigoureuses connaissances des langues.

La première partie de l'étude du texte parallèle polonais-italien du drame Przed sklepem jubilera consistait en une observation des éléments lexicaux et phraséologiques présents dans le texte original polonais et qui ne sont pas représentés " littéralement» dans le texte italien. Cela a permis de caractériser la valeur sémantique perdue dans le processus de traduction. Dans la deuxième partie, nous avons examiné l'introduction de plusieurs unités phraséologiques dans le texte traduit en italien, alors qu'elles étaient absentes en version originale polonaise. L'analyse de ce matériel confirme les résultats des recherches précédentes sur le statut de l'unité phraséologique dans le texte cible de la traduction littéraire qui montrent que les unités phraséologiques constituent souvent une "valeur ajoutée " du texte de traduction. Aussi bien des traces de pertes sémantiques que des éléments ajoutés indiquent clairement un rôle créatif du traducteur littéraire 


\section{BIBLIOGRAPHIE}

Bally, Charles. Traité de stylistique française, vol. 1, Carl Winter's Universitätsbuchhandlung, 1909.

Bogusławski, Andrzej. «Uwagi o pracy nad frazeologią ». Studia z polskiej leksykografii współczesnej, vol. 3, dir. Zofia Saloni, Dział Wydawnictw Filii UW, 1989, pp. 7-30.

Delisle Jean, Hannelore Lee-Jahnke, et Monique C. Cormier, direction. Terminologie de la traduction. John Benjamins Publishing Company, 1999.

Jean Paul II. Entrez dans l'Espérance. Avec la collaboration de Vittorio Messori. Plon/Mame, 1994.

Jezierska, Beata. Frazeologizmy w polskich przekładach wspótczesnej prozy francuskiej. Wydawnictwo „Poznańskie Studia Polonistyczne”, 2016.

Jezierska, Beata. «Status of phraseological units in the target text of translation » [article en cours de publication].

Komorowska, Agnieszka. «Literackie próby zgłębienia człowieczeństwa w trzech dramatach Karola Wojtyły: Brat naszego Boga, Przed sklepem jubilera i Promieniowanie ojcostwa ». Czlowiek $w$ refleksji Karola Wojtyly - Jana Pawła II. Wybrane aspekty adekwatnej antropologii, dir. Anna Różyło et ks. Mariusz Sztaba, Wydawnictwo KUL, 2014, pp. 215-223.

Kopeć, Urszula. «Językowo-kulturowy obraz miłości małżeńskiej i rodzinnej. Karol Wojtyła Przed sklepem jubilera ». Literatura wspótczesna w edukacji polonistycznej. Interpretacje - wartości - konteksty, t. 2, dir. Urszula Kopeć, Wydawnictwo Uniwersytetu Rzeszowskiego, 2017, pp. 29-40.

Legeżyńska, Anna. «Tłumacz czyli drugi autor». Miejsca wspólne. Szkice o komunikacji literackiej $i$ artystycznej, dir. Edward Balcerzan et Seweryna Wysłouch, PWN, 1985, pp. 160-181.

Pieńkos, Jerzy. Podstawy przekładoznawstwa. Od teorii do praktyki. Zakamycze, 2003.

Sawicki, Stefan. « Trylogia dramatyczna Karola Wojtyły ». Dramat i teatr religijny w Polsce, dir. Irena Sławińska et Wojciech Kaczmarek, Towarzystwo Naukowe KUL, pp.448-456.

\section{TEXTES SOURCES ET DICTIONNAIRES}

KW-IT : Wojtyła, Karol - Jawień, Andrzej. La bottega dell'orefice. Meditazioni sul sacramento del matrimonio che di tanto in tanto si trasformano in dramma, trad. di Aleksandra Kurczab e Jerzy Pomianowski, con la collaborazione di Siro Angeli, commento di Jerzy Pomianowski, Libreria Editrice Vaticana, 1979.

KW-FR : Wojtyła Karol - Jawień Andrzej. La Boutique de l'Orfèvre. Méditations sur le sacrament du mariage se transformant, de temps à l'autre, en dramme, texte français de Koukou Chanska, préface de Jean- Louis Barrault, avant propos de Jean Offredo. Editions Cana, 1979.

KW-PL : Wojtyła, Karol. Przed sklepem jubilera. Medytacja o sakramencie małżeństwa przechodzaca chwilami w dramat. Wydawnictwo TUM, 2001. (Przedruk z: Karol Wojtyła, Poezje i dramaty. Wydawnictwo Znak 1980.

DEL : Dictionnaire des expressions et locutions. Deuxième édition, dir. Alain Rey et Sophie Chantreau, Dictionnaires LE ROBERT, 2006.

COR : Dizionario dei modi di dire della lingua italiana, dir. Monica Quartu et Elena Rossi, Hoepli, 2012. dizionari.corriere.it/dizionario-modi-di-dire/. Consulté 30.11. 2020. 
ETYM : Słownik etymologiczny języka polskiego, dir. Aleksander Brückner, Krakowska Spółka Wydawnicza, 1927. pl.wikisource.org/wiki/S\%C5\%82ownik_etymologiczny_j\%C4 $\% 99$ zyka_polskiego. Consulté 30.11.2020.

PSFBiL : Popularny stownik frazeologiczny, dir. Stanisław Bąba et Jarosław Liberek, Warszawa: Wydawnictwo Naukowe PWN, 2009 [CD].

WSF: Wielki słownik frazeologiczny PWN z przysłowiami, dir. Anna Kłosińska, Elżbieta Sobol et Anna Stankiewicz, Wydawnictwo Naukowe PWN, 2005.

SJP : $\quad$ Stownik języka polskiego PWN. sjp.pwn.pl/. Consulté 30.11.2020.

SJPS : Słownik języka polskiego, dir. Witold Doroszewski, PWN, (1958-1969) . En ligne: http://sjpd.pwn.pl/. Consulté 30.11.2020.

TRE : Il Vocabolario Treccani. www.treccani.it/vocabolario/. Consulté 30. 11.2020.

WSJP : Wspótczesny stownik języka polskiego, dir. Bogusław Dunaj, Langenscheidt, 2007.

WSWO : Wielki słownik wyrazów obcych PWN, dir. Mirosław Bańko, Wydawnictwo Naukowe PWN, 2003.

\section{LA VALEUR SÉMANTIQUE AJOUTÉE ET PERDUE DANS LA TRADUCTION EN ITALIEN DU DRAME POLONAIS PRZED SKLEPEM JUBILERA DE KAROL WOJTYŁA}

$$
\text { Rés u mé }
$$

L'objectif de l'article consiste à décrire un phénomène de valeur ajoutée et perdue au niveau lexical et sémantique dans la traduction littéraire. L'étude est basée sur l'analyse du texte parallèle polonais-italien du drame Przed sklepem jubilera de Karol Wojtyła. La comparaison du texte original polonais avec sa traduction italienne a permis de distinguer des nuances sémantiques perdues au niveau lexical de la traduction. En revanche, le résultat de l'opération inverse (comparaison du texte d'arrivée en italien avec le texte de départ en polonais) prouve la présence de nombreux éléments lexicaux « ajoutés » dans le texte cible. Ce sont surtout des combinaisons d'un certain degré du figement, autrement dit, des unités phraséologiques. Des traces de pertes sémantiques aussi bien que des éléments ajoutés indiquent clairement un rôle créatif du traducteur littéraire qui devient ainsi le « deuxième auteur » d'un texte.

Mots-clé: traduction littéraire; choix lexicaux; unités phraséologiques; texte parralel.

\section{WARTOŚĆ SEMANTYCZNA DODANA I UTRACONA WE WŁOSKIM TŁUMACZENIU POLSKIEGO DRAMATU PRZED SKLEPEM JUBILERA KAROLA WOJTYŁY}

$$
\text { Streszczenie }
$$

Celem artykułu jest opisanie zjawiska wartości dodanej i utraconej na poziomie leksykalnym i semantycznym w tłumaczeniu literackim. Podstawę badania stanowi analiza tekstu równoległego polsko-włoskiego obejmującego dramat Przed sklepem jubilera Karola Wojtyły. Porównanie tekstu polskiego oryginału z jego włoskim tłumaczeniem pozwoliło na wyróżnienie pewnych utraconych na poziomie leksykalnym odcieni semantycznych, natomiast działanie odwrotne (porównanie tekstu docelowego po włosku z tekstem wyjściowym po polsku) wykazało obecność wielu elementów leksykalnych „,dodanych” w tekście finalnym - mowa szczególnie o kombina- 
cjach wielowyrazowych o różnym stopniu idiomatyczności, to znaczy o związkach frazeologicznych. Zarówno ślady strat semantycznych, jak i wszelkie elementy dodane wskazują jasno na kreatywną rolę tłumacza literackiego, który w ten sposób staje się „drugim autorem” tekstu.

Słowa kluczowe: przekład literacki; wybory leksykalne; frazeologizmy; tekst równoległy. 